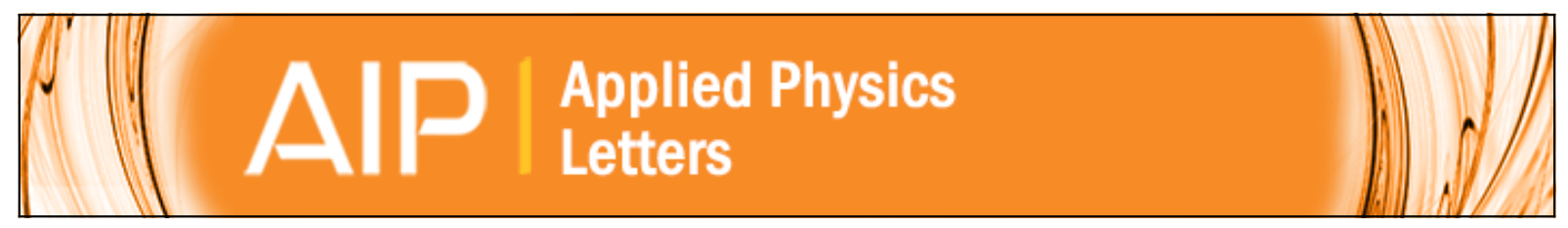

Metalorganic vapor-phase epitaxial growth of vertically well-aligned $\mathrm{ZnO}$ nanorods

W. I. Park, D. H. Kim, S.-W. Jung, and Gyu-Chul Yi

Citation: Applied Physics Letters 80, 4232 (2002); doi: 10.1063/1.1482800

View online: http://dx.doi.org/10.1063/1.1482800

View Table of Contents: http://scitation.aip.org/content/aip/journal/apl/80/22?ver=pdfcov

Published by the AIP Publishing

Articles you may be interested in

Evidence for low density of nonradiative defects in $\mathrm{ZnO}$ nanowires grown by metal organic vapor-phase epitaxy Appl. Phys. Lett. 91, 143120 (2007); 10.1063/1.2794790

Synthesis and optical properties of well-aligned $\mathrm{ZnO}$ nanorod array on an undoped $\mathrm{ZnO}$ film Appl. Phys. Lett. 86, 031909 (2005); 10.1063/1.1854737

Optical and structural analysis of $\mathrm{ZnCdO}$ layers grown by metalorganic vapor-phase epitaxy Appl. Phys. Lett. 83, 3290 (2003); 10.1063/1.1620674

Excitonic emissions observed in $\mathrm{ZnO}$ single crystal nanorods Appl. Phys. Lett. 82, 964 (2003); 10.1063/1.1544437

Time-resolved and time-integrated photoluminescence in $\mathrm{ZnO}$ epilayers grown on Al $2 \mathrm{O} 3$ (0001) by metalorganic vapor phase epitaxy

Appl. Phys. Lett. 80, 1924 (2002); 10.1063/1.1461051

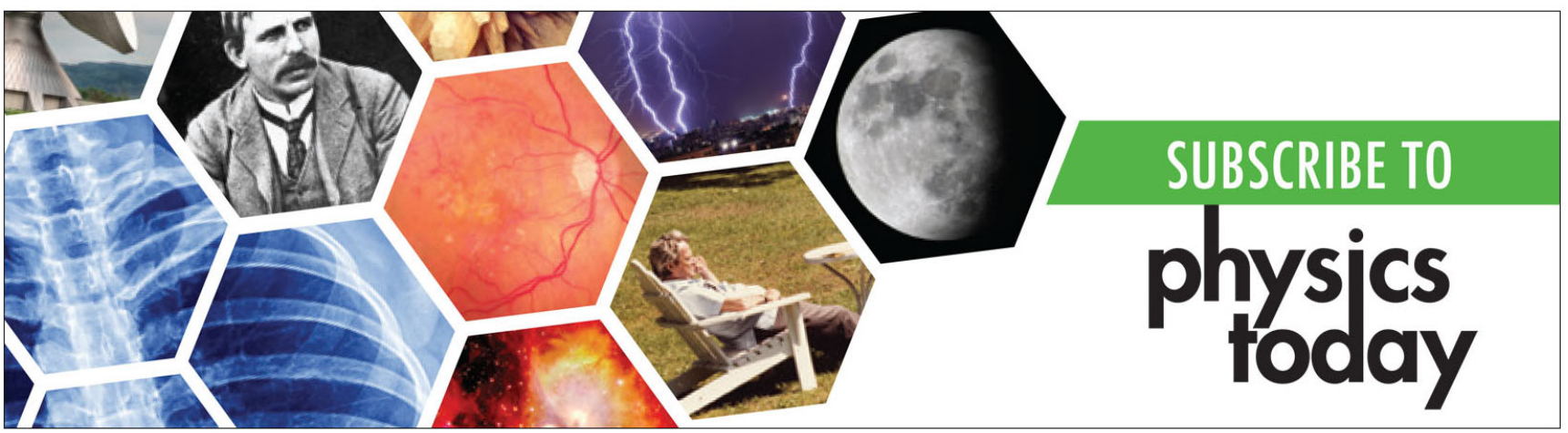




\title{
Metalorganic vapor-phase epitaxial growth of vertically well-aligned ZnO nanorods
}

\author{
W. I. Park, D. H. Kim, S.-W. Jung, and Gyu-Chul Yia) \\ Department of Materials Science and Engineering, Pohang University of Science and Technology \\ (POSTECH), Pohang 790-784, Korea
}

(Received 17 September 2001; accepted for publication 4 April 2002)

\begin{abstract}
We report metalorganic vapor-phase epitaxial growth and structural and photoluminescent characteristics of $\mathrm{ZnO}$ nanorods. The nanorods were grown on $\mathrm{Al}_{2} \mathrm{O}_{3}(00 \cdot 1)$ substrates at $400{ }^{\circ} \mathrm{C}$ without employing any metal catalysts usually needed in other methods. Electron microscopy revealed that nanorods with uniform distributions in their diameters, lengths, and densities were grown vertically from the substrates. The mean diameter of the nanorods is as narrow as $25 \mathrm{~nm}$. In addition, x-ray diffraction measurements clearly show that $\mathrm{ZnO}$ nanorods were grown epitaxially with homogeneous in-plane alignment as well as a $c$-axis orientation. More importantly, from photoluminescence spectra of the nanorods strong and narrow excitonic emission and extremely weak deep level emission were observed, indicating that the nanorods are of high optical quality. (C) 2002 American Institute of Physics. [DOI: 10.1063/1.1482800]
\end{abstract}

One-dimensional semiconductor nanowires and nanorods have attracted increasing interest due to their physical properties and diversity for potential electronic and photonic device applications. ${ }^{1,2}$ Many nanowires including Si, Ge, InP, $\mathrm{GaAs}, \mathrm{GaN}$, and $\mathrm{ZnO}$ have been fabricated for applications since $\mathrm{Si}$ submicron whiskers were grown using a catalysisassisted vapor-liquid-solid (VLS) growth method. ${ }^{3-5}$ In VLS nanowire growth, impurities act as catalysts and play an essential role in forming liquid alloy droplets for deposition on a preferred site. ${ }^{5}$ During growth, the catalyst might be incorporated into nanowires, and generate unintentional defect levels, e.g., Au used as a typical catalyst in VLS growth is well known to be a trap center in $\mathrm{Si}^{6}{ }^{6}$ However, even low defect concentrations affect physical properties of semiconductors, and, hence, unintentionally doped impurities are detrimental to device fabrication. In this letter, we introduce metalorganic vapor-phase epitaxy (MOVPE) as a nanorod growth method which uses no metal catalyst. ${ }^{7}$

In addition to the control of impurities, heteroepitaxial growth and thickness control in the nanometer range are required for fabrication of sophisticated electronic and photonic devices. These are accomplished using advanced epitaxial growth techniques such as MOVPE and molecular beam epitaxy. ${ }^{8,9}$ MOVPE is of particular interest since it has many advantages such as the feasibility of large area growth as well as simple and accurate doping and thickness control. Although this technique has widely been used for epitaxial film growth, it has rarely been employed for the preparation of one-dimensional nanorods. ${ }^{10}$ In this letter, we report MOVPE growth of $\mathrm{ZnO}$ nanorods, which requires no metal catalysts. The nanorod growth temperature was as low as $400{ }^{\circ} \mathrm{C}$. More importantly, nanorods grown in the course of this research are vertically well-aligned and exhibit uniform thickness and length distributions, which are very useful for many device applications. ${ }^{2,11}$

\footnotetext{
a) Author to whom correspondence should be addressed; electronic mail: gcyi@postech.ac.kr
}

$\mathrm{ZnO}$ nanorods were grown on $\mathrm{Al}_{2} \mathrm{O}_{3}(00 \cdot 1)$ substrates using a low pressure MOVPE system. For $\mathrm{ZnO}$ nanorod growth, diethylzinc (DEZn) and oxygen were employed as the reactants and argon was used as a carrier gas. Oxygen and DEZn flow rates were in the range of 20-100 and 0.5-5 sccm at a DEZn bubbler temperature of $-15-0{ }^{\circ} \mathrm{C}$, respectively. A typical growth temperature was in the range of 400-500 ${ }^{\circ}$ C. ${ }^{12,13}$ Prior to $\mathrm{ZnO}$ nanorod growth, very thin $\mathrm{ZnO}$ buffer layers were grown at a low temperature. In this growth, no metal catalyst is coated on the substrates.

The crystal structure of $\mathrm{ZnO}$ nanorods was investigated by $\mathrm{x}$-ray diffraction (XRD) including $\theta-2 \theta$ scan, $\theta$-rocking curve, and azimuthal $(\phi)$ scan measurements. The $\theta$-rocking curve and pole figure measurements were carried out for the $(00 \cdot 2)$ and $(10 \cdot 2)$ reflections of as-grown $\mathrm{ZnO}$ nanorods, respectively.

For optical characterization of the nanorods, photoluminescence (PL) spectroscopy was employed. The PL measurements were performed at room temperature with an optical resolution of $0.02-0.1 \mathrm{~nm}$, and the $325 \mathrm{~nm}$ line of a continuous wave $\mathrm{He}-\mathrm{Cd}$ laser was used as the excitation source. Details of the PL measurements have previously been reported. ${ }^{14}$

Field emission scanning electron microscopy (FE-SEM) clearly revealed the general morphology of the $\mathrm{ZnO}$ nanorods. As shown in Figs. 1(a) and 1(b), the images of $\mathrm{ZnO}$ nanorods grown for $1 \mathrm{~h}$ exhibited mean diameters and lengths of $\sim 25$ and $800 \mathrm{~nm}$, respectively. The diameter of nanorods obtained by MOVPE is somewhat smaller than the typical diameters of 50-100 $\mathrm{nm}$ for those prepared by other deposition methods. ${ }^{2,15,16}$ Furthermore, the $\mathrm{ZnO}$ nanorods are well aligned vertically, showing uniformity in their diameters, lengths, and densities.

As shown in Fig. 1(c), hexagon-shaped pyramids were observed at the ends of MOVPE-grown $\mathrm{ZnO}$ nanorods. This observation suggests that the nanorods were grown by a noncatalysis growth mechanism. In the nanorod growth process using the catalysis-assisted VLS mechanism, nanosized 

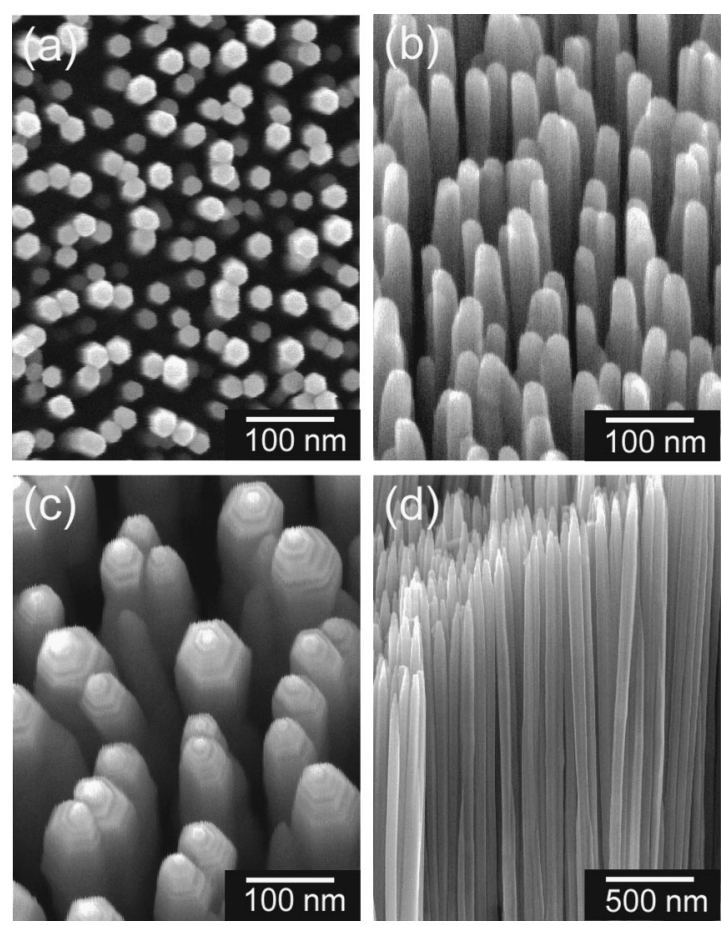

FIG. 1. FE-SEM (a) plan-view and (b) tilted images of $\mathrm{ZnO}$ nanorods with a mean diameter of $\sim 25 \mathrm{~nm}$ and (c) tilted and (d) cross-sectional images of $\mathrm{ZnO}$ nanorods with a mean diameter of $\sim 70 \mathrm{~nm}$. In Fig. 1(c), hexagonshaped pyramids with flat terraces and steps are shown at the ends of nanorods.

metal clusters have a critical role as a catalyst in forming liquid droplets that adsorb the gas-phase reactants where nanorod growth occurs. Hence, metallic nanoparticles are commonly observed at the end of nanorods grown by the catalysis-assisted VLS method. ${ }^{3-5,10,16}$ For MOVPE-grown nanorods, however, flat terraces and steps on the hexagonshaped ends are clearly shown in Fig. 1(c), which results from the layer-by-layer growth mode on the top layers of the nanorods. This is consistent with the previous report on similar hexagon-shape pyramids of microcrystalline grains in $\mathrm{ZnO}$ epilayers. ${ }^{17} \mathrm{In}$ addition, the $\mathrm{ZnO}$ nanorod tips were examined using energy dispersive $\mathrm{x}$-ray (EDX) spectroscopy in the FE-SEM chamber, which confirmed that there was no metal clusters present. It is also noted that no impurity in the nanorods was observed within the detection limit of the EDX spectroscopy. These results exclude the possibility that the nanorods were grown by the catalysis-assisted growth mechanism.

The crystal structure and orientation of the as-grown nanorods were investigated measuring XRD. From the XRD $\theta-2 \theta$ scan data of the $\mathrm{ZnO}$ nanorods [Fig. 2(a)] only two $\mathrm{ZnO}(00 \cdot 2)$ and $(00 \cdot 4)$ peaks were observed at $34.32^{\circ}$ and $72.59^{\circ}$, respectively. The observation of only $(00 \cdot l)$ peaks indicates that nanorods were grown with a $c$-axis orientation. However, many $\mathrm{ZnO}$ nanorods prepared by other methods have been polycrystalline or grown with random orientations. ${ }^{15,16,18}$ Meanwhile, XRD $\theta$-rocking curve measurements were also performed to investigate the degree of alignment to the normal direction of the surface. Figure 2(b) shows the rocking curve data of the sample shown in Fig. 1(a), indicating a full width at half maximum (FWHM) value of $0.6^{\circ}$. Typically, $\mathrm{ZnO}$ nanorods grown on $\mathrm{Al}_{2} \mathrm{O}_{3}(00 \cdot 1)$ (a)
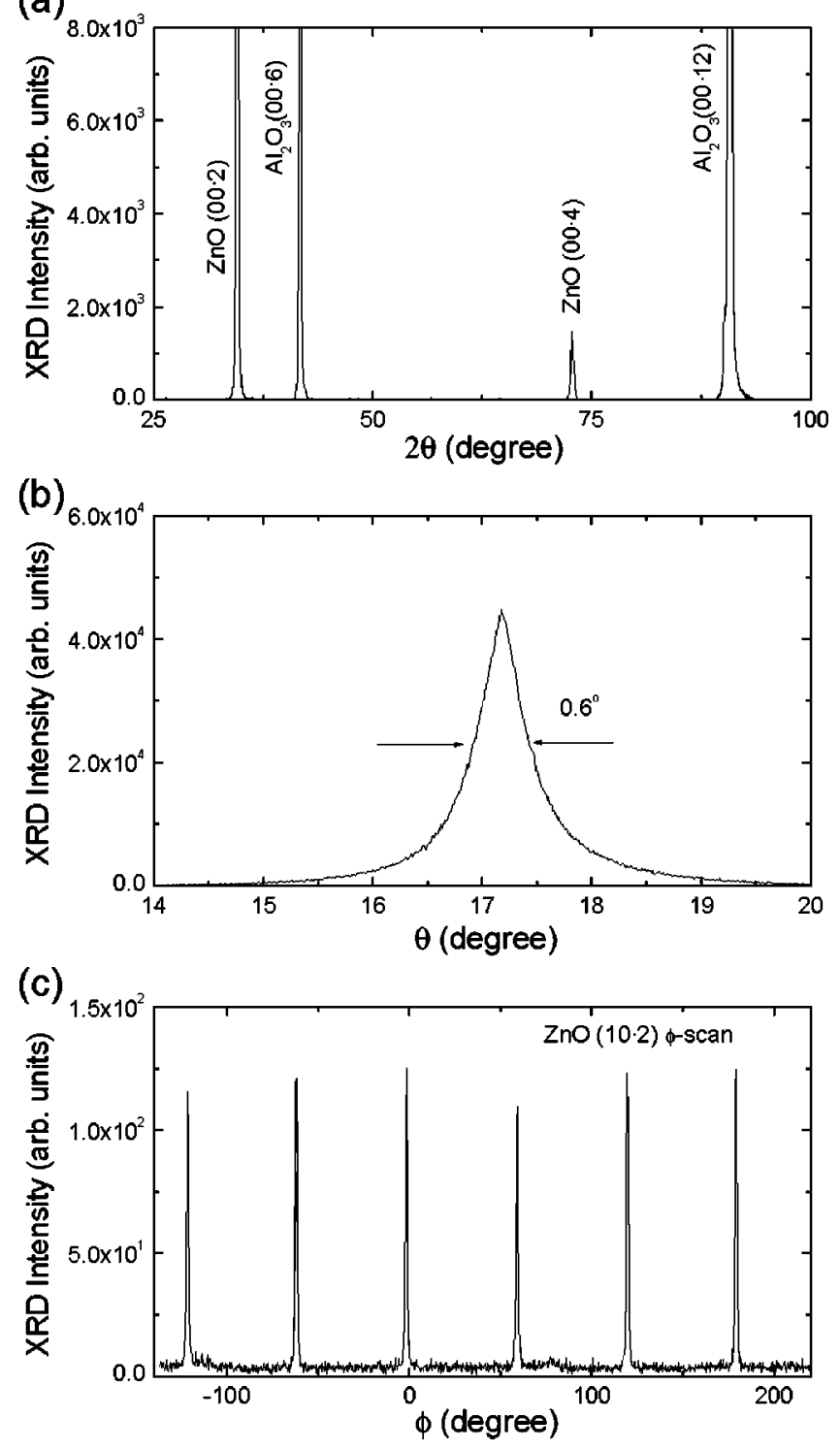

FIG. 2. XRD (a) $\theta-2 \theta$ scan, (b) rocking curve, and (c) azimuthal ( $\phi$ ) scan measurement results of $\mathrm{ZnO}$ nanorods. From the XRD $\theta-2 \theta$ scan data, only two peaks are shown at $34.32^{\circ}$ and $72.59^{\circ}$ which correspond to $\mathrm{ZnO}(00 \cdot 2)$ and $(00.4)$ peaks, respectively. The rocking curve also shows a FWHM value of $0.6^{\circ}$. A six-fold symmetry in $\phi$-scan data is also observed, indicating in-plane alignment of the nanorods.

substrates show a narrow FWHM in the range of $0.1^{\circ}-1^{\circ}$, depending on the growth conditions. The narrow FWHM in the XRD rocking curves imply that the $c$ axes of nanorods are well oriented along the normal direction of the substrate surface. Furthermore, Fig. 2(c) shows a six-fold rotational symmetry in the azimuthal scan. These results clearly indicate that the $\mathrm{ZnO}$ nanorods were epitaxially grown with homogeneous in-plane alignment as well as $c$-axis orientation. The in-plane alignment of nanorods is confirmed from the orientation of the facets in the FE-SEM image [Fig. 1(a)].

Figure 3 shows the FE-SEM images of $\mathrm{ZnO}$ nanorods at early stages of the nanorod growth. The nanorods exhibited the mean diameters of 13 and $16 \mathrm{~nm}$ after growth for 6 and $10 \mathrm{~min}$, respectively. After further growth for $1 \mathrm{~h}$, however, the nanorods exhibited only a little increase in the diameter but significant increase in the length. This result strongly suggests that the nanorods are grown on $\mathrm{ZnO}$ nuclei and the 


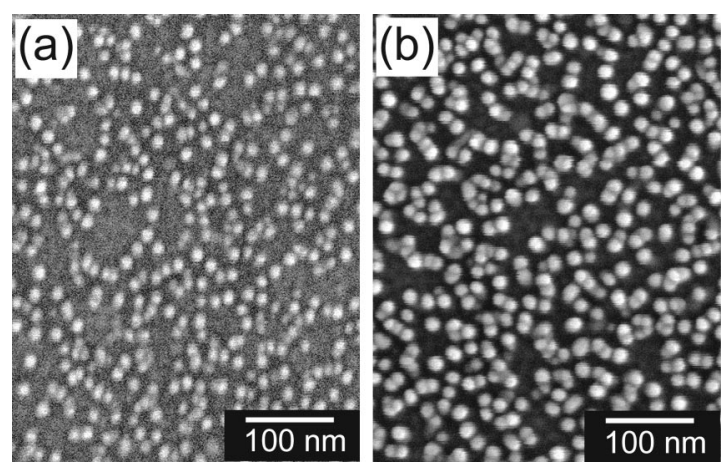

FIG. 3. FE-SEM images of $\mathrm{ZnO}$ nanorods grown for (a) 6 and (b) $10 \mathrm{~min}$. The nanorods grown for 6 and 10 min show the mean diameter of 13 and 16 $\mathrm{nm}$, respectively. Nanorods grown in this method show only a little increase in the diameters with increasing growth time.

nanorod formation results from the higher growth rate along the $c$-axis direction. However, it is noted that the spacing of the nanorods becomes larger with increasing growth time, which might result from a "crowding effect" of closely spaced that strongly favors upward growth.

As for the mechanism of the nanorod growth, nucleation at the initial stage might have a crucial role in both the vertical and in-plane alignments of the nanorods. Since the metal catalyst is not used for the nanorod growth in this research, the nucleation can occur at any sites on the substrate. During the formation of nuclei, $\mathrm{ZnO}$ nuclei are grown with an epitaxial relationship with $\mathrm{Al}_{2} \mathrm{O}_{3}(00 \cdot 1)$ since $\mathrm{ZnO}$ and $\mathrm{Al}_{2} \mathrm{O}_{3}$ are lattice matched. ${ }^{12}$ This argument is strongly supported by the fact that FWHM values in XRD rocking curves of the nanorods were decreased by the use of low temperature $\mathrm{ZnO}$ buffer layer, presumably due to enhanced epitaxy of $\mathrm{ZnO}$ nuclei.

The optical properties of the $\mathrm{ZnO}$ nanorods were investigated by PL spectroscopy. From room temperature PL spectrum of $\mathrm{ZnO}$ nanorods in Fig. 4, the dominant peak was observed at $3.29 \mathrm{eV}$, which is attributed to the free exciton peak. $^{12-14}$ The FWHM of the free exciton peak was as narrow as $90 \mathrm{meV}$. This result indicates that a PL peak shift due to the quantum confinement effect is not observed in these $\mathrm{ZnO}$ nanorods, presumably due to the large diameters of nanorods exceeding $20 \mathrm{~nm}$. Meanwhile, the deep level green emission associated with point defects commonly observed in $\mathrm{ZnO}$ epilayers was found to be extremely weak compared with the near band edge emission. The weak deep level emission might be related to the low impurity concentration determined by EDX spectroscopy. The strong and sharp excitonic emission and low deep level emission indicate that the $\mathrm{ZnO}$ nanorods are of excellent optical quality, comparable to the $\mathrm{ZnO}$ epilayers.

In conclusion, MOVPE demonstrated the epitaxial growth of vertically aligned $\mathrm{ZnO}$ nanorods at a low temperature of $400{ }^{\circ} \mathrm{C}$. In this process, no metal catalyst was used. The FE-SEM images showed uniform thickness and length distributions. Furthermore, the nanorods are of high crystal-

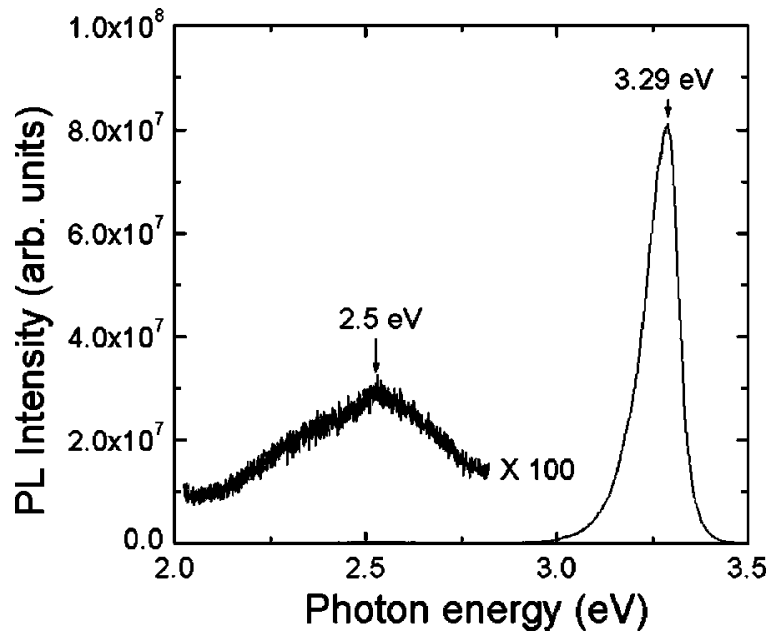

FIG. 4. PL spectrum of $\mathrm{ZnO}$ nanorods measured at room temperature. A dominant free exciton peak at room temperature was observed at $3.29 \mathrm{eV}$, indicating no peak shift due to the quantum confinement. Deep level emission is also shown to be extremely weak even at room temperature.

linity and excellent optical quality. XRD measurements exhibited both in-plane aligned and $c$-axis oriented growth of nanorods. From the PL spectra measured at room temperature, a narrow and strong emission peak was observed at $3.29 \mathrm{eV}$ with a very weak deep level emission.

This work was supported by the KOSEF through the Regional University Research Program. Extensive use of the facilities at POSTECH is acknowledged.

${ }^{1}$ X. Duan, Y. Huang, Y. Cui, J. Wang, and C. M. Lieber, Nature (London) 409, 66 (2001).

${ }^{2}$ M. H. Huang, S. Mao, H. Feick, H. Yan, Y. Wu, H. Kind, E. Weber, R. Russo, and P. Yang, Science 292, 1897 (2001).

${ }^{3}$ X. Duan and C. M. Lieber, Adv. Mater. 12, 298 (2000).

${ }^{4}$ J. Hu, T. W. Odom, and C. M. Lieber, Acc. Chem. Res. 32, 435 (1999).

${ }^{5}$ R. S. Wagner and W. C. Ellis, Appl. Phys. Lett. 4, 89 (1964).

${ }^{6}$ S. Braun and H. G. Grimmeiss, J. Appl. Phys. 45, 2658 (1974).

${ }^{7}$ M. He, I. Minus, P. Zhou, S. N. Mohammed, J. B. Halpern, R. Jacobs, W. L. Sarney, L. Salamanca-Riba, and R. D. Vispute, Appl. Phys. Lett. 77, 3731 (2000).

${ }^{8}$ G. B. Stringfellow, Organometallic Vapor-Phase Epitaxy: Theory and Practice (Academic, Boston, 1989).

${ }^{9}$ A. Y. Cho and I. Hayashi, J. Appl. Phys. 42, 4422 (1971).

${ }^{10}$ M. Yazawa, M. Koguchi, A. Muto, M. Ozawa, and K. Hiruma, Appl. Phys. Lett. 61, 2051 (1992).

${ }^{11}$ S. Fan, M. G. Chapline, N. R. Franklin, T. W. Tombler, A. M. Cassell, and H. Dai, Science 283, 512 (1999).

${ }^{12}$ W. I. Park, S.-J. An, G.-C. Yi, and H. M. Jang, J. Mater. Res. 16, 1358 (2001).

${ }^{13}$ W. I. Park, G.-C. Yi, and H. M. Jang, Appl. Phys. Lett. 79, 2022 (2001).

${ }^{14}$ W. I. Park and G.-C. Yi, J. Electron. Mater. 30, L32 (2001).

${ }^{15}$ Y. C. Kong, D. P. Yu, B. Zhang, W. Fang, and S. Q. Feng, Appl. Phys. Lett. 78, 407 (2001)

${ }^{16}$ M. H. Huang, Y. Wu, H. Feick, N. Tran, E. Weber, and P. Yang, Adv. Mater. 13, 113 (2001).

${ }^{17}$ Z. K. Tang, G. K. L. Wong, P. Yu, M. Kawasaki, A. Ohtomo, H. Koinuma, and Y. Segawa, Appl. Phys. Lett. 72, 3270 (1998); M. Kawasaki, A. Ohtomo, I. Ohkubo, H. Koinuma, Z. K. Tang, P. Yu, G. K. L. Wong, B. P. Zhang, and Y. Segawa, Mater. Sci. Eng., B 56, 239 (1998).

${ }^{18}$ Y. Li, G. W. Meng, L. D. Zhang, and F. Phillipp, Appl. Phys. Lett. 76, $2011(2000)$ 